\title{
Measuring the Physiologic Properties of Oral Lesions Receiving Fractionated Photodynamic Therapy
}

\author{
Shannon M. Gallagher-Colombo ${ }^{1}$, Harry Quon ${ }^{1 \dagger}$, Kelly M. Malloy ${ }^{2 \ddagger}$, Peter H. Ahn ${ }^{1}$, Keith A. Cengel ${ }^{1}$, \\ Charles B. Simone II', Ara A. Chalian', Bert W. O'Malley ${ }^{2}$, Gregory S. Weinstein², Timothy C. Zhu', \\ Mary E. Putt ${ }^{3}$, Jarod C. Finlay ${ }^{1 \S}$ and Theresa M. Busch*1§ \\ ${ }^{1}$ Department of Radiation Oncology, Perelman School of Medicine, University of Pennsylvania, Philadelphia, PA \\ ${ }^{2}$ Department of Otorhinolaryngology, Perelman School of Medicine, University of Pennsylvania, Philadelphia, PA \\ ${ }^{3}$ Department of Biostatistics and Epidemiology, Perelman School of Medicine, University of Pennsylvania, Philadelphia, PA \\ Received 5 November 2014, accepted 26 May 2015, DOI: 10.1111/php.12475
}

\begin{abstract}
Photodynamic therapy (PDT) can treat superficial, earlystage disease with minimal damage to underlying tissues and without cumulative dose-limiting toxicity. Treatment efficacy is affected by disease physiologic properties, but these properties are not routinely measured. We assessed diffuse reflectance spectroscopy (DRS) for the noninvasive, contact measurement of tissue hemoglobin oxygen saturation $\left(\mathrm{S}_{\mathrm{t}} \mathrm{O}_{2}\right)$ and total hemoglobin concentration ([tHb]) in the premalignant or superficial microinvasive oral lesions of patients treated with 5-aminolevulinic acid (ALA)-PDT. Patients were enrolled on a Phase 1 study of ALA-PDT that evaluated fluences of $50,100,150$ or $200 \mathrm{~J} \mathrm{~cm}^{-2}$ delivered at $100 \mathrm{~mW} \mathrm{~cm}^{-2}$. To test the feasibility of incorporating DRS measurements within the illumination period, studies were performed in patients who received fractionated (two-part) illumination that included a dark interval of 90-180 s. Using DRS, tissue oxygenation at different depths within the lesion could also be assessed. DRS could be performed concurrently with contact measurements of photosensitizer levels by fluorescence spectroscopy, but a separate noncontact fluorescence spectroscopy system provided continuous assessment of photobleaching during illumination to greater tissue depths. Results establish that the integration of DRS into PDT of early-stage oral disease is feasible, and motivates further studies to evaluate its predictive and dosimetric value.
\end{abstract}

\section{INTRODUCTION}

Photodynamic therapy (PDT) is well-suited to the treatment of early-stage head and neck malignancies as it produces a superficial effect that preserves the structure and function of underlying and adjacent tissues $(1,2)$. Head and neck squamous cell carcinoma (HNSCC) is the sixth most common cancer reported globally (3) with 5-year survival rates ranging from 36.3 to $82.7 \%$

*Corresponding author email: buschtm@mail.med.upenn.edu (Theresa M. Busch) †Current Address: Department of Radiation Oncology and Molecular Radiation Sciences, Johns Hopkins University, Baltimore, MD

¥Current Address: Department of Otolaryngology - Head and Neck Surgery,

University of Michigan School of Medicine, Ann Arbor, MI

$\S$ Senior authorship is shared between these individuals.

(C) 2015 The American Society of Photobiology depending on tumor location and stage at diagnosis (4). Multiple clinical trials and series using PDT with either temoporfin or porfimer sodium in patients with T0-T2 N0 HNSCC have been reported, demonstrating complete response rates of 85-90\% (5). Given the natural history of early HNSCC with patients typically experiencing multiple metachronous lesions, one key advantage for PDT is that, unlike radiotherapy, multiple treatment courses can be performed without accumulating additional risk for toxicity (6). On a temporary basis, however, prolonged cutaneous photosensitivity from temoporfin or porfimer sodium can be logistically challenging for patients. In this trial, we have investigated the use of 5-aminolevulinic acid (ALA), a photosensitizer-inducing prodrug that is tailored to superficial treatment applications and exhibits short duration (2 days) for cutaneous photosensitivity.

The oxygenation status of a tumor is critical to PDT response. Paradoxically, PDT can itself deplete oxygen levels through the oxygen-dependent photochemical reaction, as well as through the damage of tumor vasculature $(2,7)$. Indeed, PDT-induced vascular destruction, indicated by blood flow stasis and hemorrhage of the microvasculature, has been observed in oral squamous cell carcinomas (SCC) using side-stream dark field imaging (8). While vascular shutdown can be beneficial to tumor destruction by effectively inducing tumor ischemia, if ischemia occurs during the course of light delivery, it may impede the therapeutic effect by exacerbating photochemically induced hypoxia or by independently producing a hypoxic state. One approach to circumvent this effect is to fractionate the treatment light. This is based on the hypothesis that interruption of the light delivery may partially reverse the treatment-induced hypoxia by allowing for reperfusion of the tissue (2). The importance of maintaining sufficient oxygen levels during PDT is paramount to treatment efficacy. Our group has reported that PDT-induced changes in tumor hemoglobin oxygen saturation $\left(\mathrm{S}_{\mathrm{t}} \mathrm{O}_{2}\right)$ correlate with treatment response in preclinical investigations (9). Pogue et al. (10) reported that ALA-PDT can produce increases in $\mathrm{pO}_{2}$ during light treatment, though these increases were highly variable from mouse-to-mouse. This variability emphasizes the need to monitor oxygenation status during PDT on a case-by-case basis, as it highlights the inherent differences in tumor microenvironment across patients.

Optical methods to monitor photodynamic therapy have been developed in a variety of settings previously. The most useful of 
these generally fall into two categories: Absorption-based detection used to quantify hemodynamic parameters, and fluorescence spectroscopy or imaging used to detect photosensitizer via its fluorescence emission (11). Diffuse reflectance measurements have been widely used to characterize hemodynamics during PDT. Optical absorption measurements using compact, fiber-based probes can detect hemoglobin oxygen concentration and saturation (12) and relative changes in blood flow (13). However, diffuse reflectance technologies have difficulty detecting the photosensitizer, ALA-induced protoporphyrin IX (PpIX), due to its low absorption relative to hemoglobin. In contrast, fluorescence can be used to quantify the uptake of photosensitizer in tissue. For instance, Zhou et al. (14) demonstrated in an animal model that adjusting light dose to account for variations in pretreatment sensitizer fluorescence led to more consistent responses to PDT. The sensitizer concentration can be therefore used in an explicit dosimetric model to predict response to PDT. It is also possible to use the photobleaching of the sensitizer as an implicit dosimetric surrogate for PDT response (15). ALA-induced PpIX is highly fluorescent, and readily photobleaches due to reactions with the singlet oxygen it generates, making it an ideal candidate for the implicit dosimetry. This has been demonstrated in animal models $(16,17)$, and utilized to reduce pain in a clinical trial of PDT for superficial skin lesions (18). Recent work in our group has focused on developing combined instrumentation for reflectance and fluorescence detection compatible with clinical trials of PDT. We have implemented absorption and fluorescence detection in interstitial treatments of the human prostate $(19,20)$ and intraoperative trials in the peritoneal (21) and pleural (22) settings.

In this study, we present results pertaining to a clinical trial of ALA-PDT for premalignant and early microinvasive lesions of the oral cavity for which diffuse reflectance spectroscopy (DRS) and fluorescence spectroscopy were employed to, respectively, monitor lesion oxygenation and photosensitizer levels during PDT. Results establish that it is feasible to incorporate contactbased DRS measurements into the course of the illumination of oral lesions to determine lesion oxygenation before, during a 90$180 \mathrm{~s}$ dark interval for fractionation, and immediately after treatment. Using a contact probe, photosensitizer photobleaching can be measured concurrently with the physiologic data at discrete timepoints. In contrast, a noncontact fluorescence spectroscopy system provides the means for continuous monitoring of relative photosensitizer levels during illumination of the lesions. Data demonstrate that ALA-PDT creates measureable, patient-specific changes in the physiologic properties of oral lesions that can be analyzed in a depth-dependent manner. Early outcome data support the design of a prospective study to determine whether lesion $\mathrm{S}_{\mathrm{t}} \mathrm{O}_{2}$ is predictive of clinical outcomes in PDT of oral disease.

\section{MATERIALS AND METHODS}

Clinical trial. These studies were conducted as part of a Phase 1 prospective clinical trial of ALA-PDT for the treatment of premalignant or superficial microinvasive disease of the head and neck. The protocol was conducted under an investigator-sponsored IND with the U.S. Food and Drug Administration. This study was performed in accordance with the Declaration of Helsinki and had approval from the Institutional Review Board of the University of Pennsylvania and the University of Pennsylvania Clinical Trials Scientific Review and Monitoring Committee. Patients with biopsy-confirmed disease received an oral dose $\left(60 \mathrm{mg} \mathrm{kg}^{-1}\right)$ of
ALA (kindly supplied by DUSA Pharmaceuticals, Inc, Wilmington, MA) followed in $4-6 \mathrm{~h}$ by the administration of red light to the visualized lesion. Patients were maintained under general anesthesia for the duration of spectroscopy measurements and PDT. Light was delivered using either a microlens (MedLight SA, Ecublens, Switzerland) or with a balloon diffusing fiber (MedLight SA) that has previously been described (23). A Cerelas Series GaA1As 4W diode laser (Biolitec, Inc., East Longmeadow, MA) emitting at a peak wavelength of $632 \mathrm{~nm}(90 \%$ of the power within $629-635 \mathrm{~nm}$ ) was used as the light source. Light was delivered to the surface of the lesion at a fluence rate of $100 \mathrm{~mW} \mathrm{~cm} \mathrm{~cm}^{-2}$ as measured by a calibrated isotropic detector that was placed on the target surface at the initiation of illumination. Differences in geometry and optical properties among patients lead to variations in the amount of scattered light that contributes to the signal measured by this detector. The incident irradiance was modified to ensure that the fluence rate at the lesion surface (including light scattered from the tissue) was maintained at the prescribed fluence rate. As needed, intraoperative light shielding of the surrounding normal tissue was performed using blue surgical towels or by painting with a solution of methylene blue. Light precautions were observed for $48 \mathrm{~h}$ after ALA ingestion and included the filtering of operating room lights and the protection of the patients' exposed skin with drapes or clothing.

This was a dose escalation trial in which fluences of 50,100,150 and $200 \mathrm{~J} \mathrm{~cm}^{-2}$ were tested. At each fluence, patients received either continuous or fractionated illumination, thus the study consisted of a total of eight cohorts (each fluence, with or without fractionation). Randomization between continuous and fractionated treatment was initiated after completion of the first two cohorts, one at $50 \mathrm{~J} \mathrm{~cm}^{-2}$ with continuous illumination and one at $50 \mathrm{~J} \mathrm{~cm}^{-2}$ with fractionated light. Consequently, doses above $50 \mathrm{~J} \mathrm{~cm}^{-2}$ were designated as cohorts $3\left(100 \mathrm{~J} \mathrm{~cm}^{-2}\right), 4$ $\left(150 \mathrm{~J} \mathrm{~cm}^{-2}\right)$ and $5\left(200 \mathrm{~J} \mathrm{~cm}^{-2}\right)$, with the addition of "a" versus "b" for continuous and fractionated illumination, respectively. Three patients were treated per cohort, expanded to six patients if treatment resulted in a dose-limiting toxicity (DLT). DLTs were defined as the presence of a grade 3 or higher toxicity at 30 days after PDT; toxicities were evaluated and scored using the Cooperative Group Common Toxicity Criteria (CTCAE v.3.0). No patients were treated above the maximum tolerated dose, defined as the light dose level which resulted in no more than one DLT in six treated patients and for which the next higher dose level produced two or more DLTs in three to six treated patients. Follow up was performed on day 1, week 1, week 2-3, day 30, day 90, every 3 months for 24 months and then annually (day 0 defined as the day of PDT). At the 90 day follow-up a clinical evaluation of response was performed. Patients were scored for a complete response, which was defined as complete ablation or absence of the index lesion in the area treated with light. In the absence of a complete response, the presence of local recurrence was noted.

This report considers only those patients who received fractionated illumination. Fractionation was defined by delivery of $20 \%$ of the fluence followed by a $90-180 \mathrm{~s}$ break in illumination before resuming treatment to the full fluence (24). The length of the dark interval allowed for the integration of spectroscopy measurements when treatment was at an accessible site. In these patients, spectroscopy measurements of the lesion, as defined below, were made prior to light delivery, during the dark interval for fractionation, and after the completion of light delivery. Of note, in patient 7 , the break for fractionation occurred after $20 \%$ of $200 \mathrm{~J} \mathrm{~cm}^{-2}$ had been delivered although the patient received $150 \mathrm{~J} \mathrm{~cm}^{-2}$. This was a result of the early termination of light delivery at $150 \mathrm{~J} \mathrm{~cm}^{-2}$ of a planned dose of $200 \mathrm{~J} \mathrm{~cm}^{-2}$. Also, only one patient treated at $100 \mathrm{~J} \mathrm{~cm}^{-2}$ had a lesion that was accessible for spectroscopy measurements. Normal tissue of tongue, buccal mucosa or hard palate was measured in each patient before and after light delivery.

Instrumentation. The spectroscopic data presented here were collected by each of two independent systems. The first, the contact fluorescence and reflectance spectroscopy (CFRS) system, uses a custom-built fiberoptic based optical probe, which is placed in physical contact with the tissue being treated prior to, immediately after and during a brief interruption in illumination. This probe consists of two source optical fibers and a series of detection fibers spaced between 0.14 and $0.88 \mathrm{~cm}$ away from the first source fiber. The probe allows collection of diffuse reflectance using a white light source (Avalight; Avantes, Broomfield, CO) coupled to the first source fiber, and fluorescence spectra using a $403 \mathrm{~nm}$ excitation laser (Power Technologies, Inc., Little Rock, AK) coupled to the second source fiber. Both measurements use the same set of detection 
fibers, which are imaged by an integrated imaging spectrometer/multichannel CCD system (Inspectrum; Roper Scientific, Trenton, NJ). Data acquisition is sequential such that repeated diffuse reflectance, background and fluorescence spectra are acquired at each of multiple sample locations. Data acquisition is triggered by an electronic push-button interface that is controlled by the clinician.

The second spectroscopic measurement system, the noncontact treatment fluorescence (NCTF) system, measures fluorescence emission excited by the treatment light itself. This emission is collected by a passive, flat-cut optical fiber detector aligned to collect light from the treated area. When a microlens is used for delivery of treatment light, the detection fiber is held parallel to it. When a balloon diffuser is used, the detector fiber is fixed such that its axis is aligned with the center of the treatment field. The light collected by this fiber passes through a pair of long-pass filters (OG 570; Schott North America, Inc., Duryea, PA and RazorEdge LP02-633RU-25; Semrock, Rochester, NY) and is collected by a single-channel, TE-cooled CCD spectrometer (BTC-112E; B\&W Tek, Newark, DE). The filters block treatment light and the primary emission peak of PpIX, however, the long-wavelength secondary peak around $707 \mathrm{~nm}$ is still distinguishable from the background of off-peak laser emission and autofluorescence.

Data analysis. Data were analyzed in a combination of routines written in Matlab (The Mathworks, Natick, MA). Briefly, the absorption spectrum of tissue was assumed to be a linear combination of the absorption spectra of oxy-and deoxyhemoglobin, weighted by their concentrations. The reduced scattering spectrum was assumed to follow the form $\mu_{\mathrm{s}}^{\prime}=\mathrm{A}\left(\lambda / \lambda_{0}\right)^{-\mathrm{b}}$. This expression has been widely used to model scattering from cells (25). The diffuse reflectance is modeled using the $\mathrm{P}_{3}$-approximation-based expression developed by Hull and Foster (26), as implemented by Finlay and Foster (12). The spectra from each sourcedetector separation are fit independently. The fitting is performed in Matlab using an iterative fitting algorithm (fminsearch). The free parameters in the fit are $A, b$ and the concentrations of oxy- and deoxyhemoglobin. At each iteration, absorption and scattering spectra are constructed based on these free parameters, and a theoretical diffuse reflectance spectrum is calculated from the optical spectra. The process repeats until the calculated diffuse reflectance spectrum and the measured reflectance converge. Because the lesions being evaluated are of limited size and the tissue curvature may be extensive, only signal from the closest four fibers (with source-detector distance of $0.34 \mathrm{~cm}$ or less) are selected to be included in the analysis. From the determined quantities of oxyhemoglobin concentration $\left(\left[\mathrm{HbO}_{2}\right]\right)$ and deoxyhemoglobin concentration $([\mathrm{Hb}])$, tissue hemoglobin oxygen saturation $\left(\mathrm{S}_{\mathrm{t}} \mathrm{O}_{2}\right)$ was calculated as the ratio of $\left[\mathrm{HbO}_{2}\right]$ to total hemoglobin concentration $([\mathrm{tHb}])$, i.e. the sum of $\left[\mathrm{HbO}_{2}\right]$ and $[\mathrm{Hb}]$ and multiplied by 100 for reporting as a percentage.

Fluorescence spectra collected by the nearest fiber of the contact probe to the source are corrected for the effects of absorption and scattering by dividing by the diffuse reflectance. The corrected spectra in the range from 575 to $720 \mathrm{~nm}$ are then fit with a singular value decomposition (SVD)-based fitting algorithm (17). The basis spectra used to fit the fluorescence data shown here are tissue autofluorescence, PpIX and two fluorescent photoproducts of PpIX (17). The sensitivity of detection and the geometry of illumination may vary from patient to patient. To correct for this effect, the SVD amplitude of PpIX is divided by that of autofluorescence. Results are therefore presented as fluorescence of PpIX per unit autofluorescence.

Fluorescence excited by treatment light is fit with an SVD algorithm analogous to that used for the contact probe measurements, but in this case, only the portion of the spectrum from $660 \mathrm{~nm}$ to $730 \mathrm{~nm}$ is included in the fit. Again, the results are reported as the PpIX signal relative to autofluorescence.

Statistics. Data are summarized using means and medians. Wilcoxon signed-rank tests were used to test for the presence of depth-dependent differences in $\mathrm{S}_{\mathrm{t}} \mathrm{O}_{2}$ among detector positions. Hypothesis tests were two sided, and statistical significance was declared for $P$-values $<0.05$.

\section{RESULTS}

\section{Patient characteristics}

A total of 14 patients were treated with fractionated (two-part) light delivery. Fractionation incorporated a 90-180 s interruption

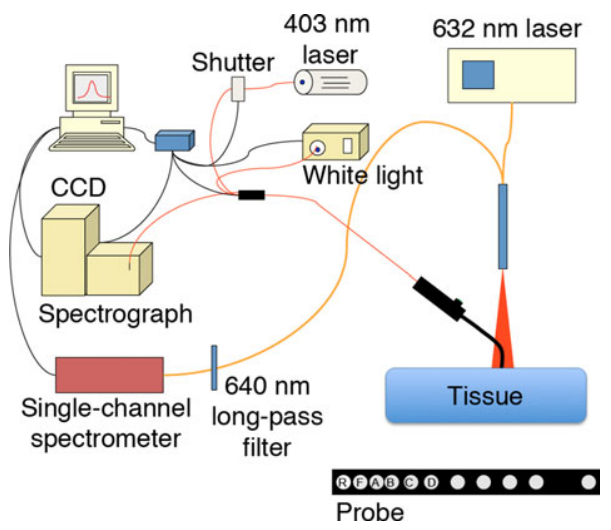

Figure 1. Schematic of the CFRS system for diffuse reflectance/fluorescence spectroscopy. Sampling with the contact probe and the laser irradiation are both shown. In reality, they are sequential. The probe face is shown in the lower right. The reflectance source (R), fluorescence source (F) and the first four detection fibers (A through D) are labeled. The remaining detection fibers were not used in this study.

of light delivery after $20 \%$ of the total fluence was delivered. Among these patients, 10 patients had lesions in the oral cavity that were accessible to measurements before light delivery, during the dark interval of fractionation and after PDT (Fig. 1). The characteristics of these patients are summarized in Table 1. Median patient age was 61 (range 34-84) and nine of the patients were female (patient 6 was male). Five patients had lesions on the oral tongue, three had lesions where the tongue meets the floor of the mouth or the alveolar ridge, and two of the patients had lesions of the buccal mucosa. Histologically, the lesions ranged from moderate dysplasia to microinvasive carcinoma for lesions on the tongue or junction of the tongue, and moderate dysplasia or carcinoma in situ for lesions of the buccal mucosa. A complete clinical response was found at 3 months after PDT in eight patients. Local recurrence occurred in patients 5 and 10 .

\section{Diffuse reflectance spectroscopy to measure physiologic parameters}

Physiologic parameters assessed in this study were obtained from values for $\left[\mathrm{HbO}_{2}\right]$ and $[\mathrm{Hb}]$ that were taken from fits to diffuse reflectance spectra. A typical set of diffuse reflectance spectra and their fitting is shown in Fig. 2a,b, respectively. In this figure, the best-fit theoretical curve is plotted along with the measured data. For comparison, the components of the absorption in this spectral range are also shown. Because it is impossible to generate a theoretical reflectance curve in the absence of scattering, these curves are generated assuming the best-fit scattering and absorption composed of only one component $\left(\mathrm{Hb}\right.$ or $\left.\mathrm{HbO}_{2}\right)$. The agreement between the theoretical and measured data indicates that the scattering model and two component absorbers are sufficient to model the in vivo optical properties.

Tissue oxygenation, calculated as $\mathrm{S}_{\mathrm{t}} \mathrm{O}_{2}$, and blood volume, calculated as [tHb], were determined from the fitted measurements of $\left[\mathrm{HbO}_{2}\right]$ and $[\mathrm{Hb}]$. Figure $3 \mathrm{a}$ depicts lesion $\mathrm{S}_{\mathrm{t}} \mathrm{O}_{2}$ levels at baseline, during the dark interval for fractionation, and after the completion of light delivery in each patient. In Fig. 3b, values for $[\mathrm{tHb}]$ are shown. For both figures, data are plotted as a function of the delivered light fluence, and, for simplicity, individual panels depict patients treated at different light fluences. 
Table 1. Patient characteristics.

\begin{tabular}{lllccc}
\hline Patient no. & \multicolumn{1}{c}{ Histology } & \multicolumn{1}{c}{ Site } & Fluence $\left(\mathrm{J} \mathrm{cm}^{-2}\right)$ & PpIX fluorescence (a.u.) & Response at 3 months* \\
\hline 1 & High/severe dysplasia & Tongue & 50 & 93 & CR \\
2 & Carcinoma in situ & Tongue & 50 & 11 & CR \\
3 & High/severe dysplasia & Junction of tongue/floor of mouth & 50 & 17 & CR \\
4 & Moderate dysplasia & Junction of tongue/alveolar ridge & 100 & 18 & CR \\
5 & Moderate dysplasia & Tongue & 150 & 51 & LR \\
6 & Microinvasive carcinoma & Tongue & 150 & 22 & CR \\
7 & Moderate dysplasia & Tongue & 200 & 52 & CR \\
8 & Carcinoma in situ & Buccal mucosa & 200 & 133 & CR \\
9 & Moderate dysplasia & Buccal mucosa & 200 & 85 & CR \\
10 & High/severe dysplasia & Junction of tongue/floor of mouth & &
\end{tabular}

*Response at 3 months is characterized as complete response (CR) or local recurrence (LR).

a

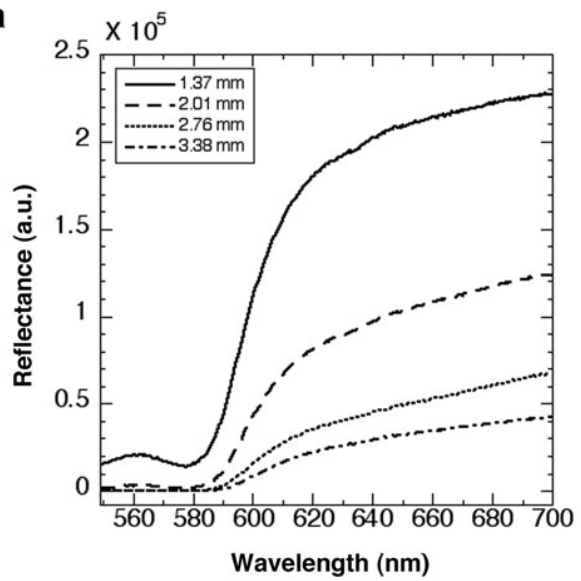

b

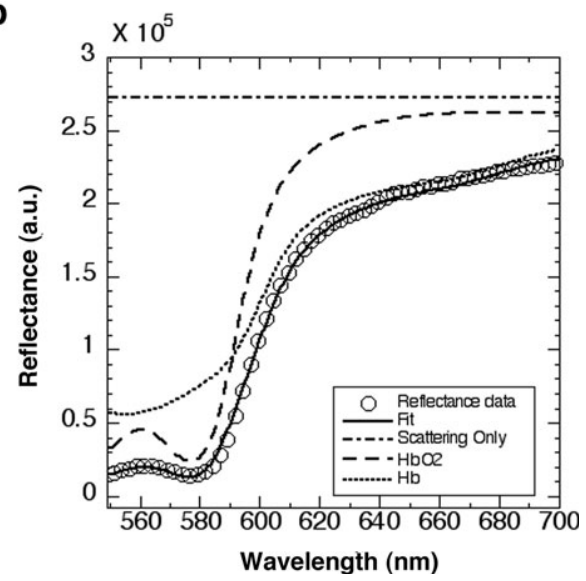

Figure 2. (a) Typical diffuse reflectance spectra measured by the CFRS system at different source-detector separations. (b) Fitting of the $1.37 \mathrm{~mm}$ spectrum. The spectrum considering negligible scattering, and those considering only the absorption contributions of oxy- and deoxyhemoglobin are shown, along with the measured data and the fitting result.

At baseline, $\mathrm{S}_{\mathrm{t}} \mathrm{O}_{2}$ values clustered between $\sim 45 \%$ and $65 \%$ in the lesions of most patients. However, patient 9 had a pre-PDT $\mathrm{S}_{\mathrm{t}} \mathrm{O}_{2}$ value of $16 \%$ and patients 5 and 10 had very low values of $<10 \%$. Treatment-induced changes in lesion oxygenation were detected in all patients, suggesting that PDT can alter the physiologic properties of even very superficial disease. At PDT conclusion, lesion $\mathrm{S}_{\mathrm{t}} \mathrm{O}_{2}$ was $>35 \%$ in all but patients 5 and 10 . In general, the magnitude of PDT effect on lesion $\mathrm{S}_{\mathrm{t}} \mathrm{O}_{2}$ was similar among patients, although the direction of the change did vary.
Again, patient 10 was an exception; in this patient, a large increase in $\mathrm{S}_{\mathrm{t}} \mathrm{O}_{2}$ was detected during the break in light delivery for fractionation. Yet, irrespective of this increase at fractionation, at the conclusion of PDT, lesion $\mathrm{S}_{\mathrm{t}} \mathrm{O}_{2}$ returned to a value that was similar to its low baseline level. In this regard, it is interesting to observe that the two patients (5 and 10) with the poorest oxygenated disease at baseline and at the conclusion of PDT were those who failed to achieve a complete response at 3 months.

In contrast to its effect on $\mathrm{S}_{\mathrm{t}} \mathrm{O}_{2}$, PDT tended to produce larger and more variable changes in blood volume. In considering these data, it is important to note that DRS measurements were made very quickly (within seconds of the light being turned off) during the dark interval of fractionation. In contrast, at the conclusion of therapy, the illumination setup and shielding was removed prior to beginning DRS measurements. This led to a potential lapse of several minutes between therapy conclusion and physiologic measurements. These differences in the delay between termination of illumination and DRS measurements could lead to inconsistencies in physiologic properties measured at a given fluence, as a function of whether this fluence fell during the dark interval of fractionation or at the conclusion of PDT. Measures of [tHb] are likely particularly sensitive to this caveat, as PDT can lead to rapid changes in blood flow (27).

\section{Depth dependencies in lesion oxygenation are measureable}

The DRS probe includes multiple detectors at different distances from the source light fiber. This design provides for the depthaveraged assessment of tissue physiology when data are averaged across all of the detector fibers, as was done for the values reported above. Alternatively, depth-dependent tissue oxygenation can be obtained if the data are segregated as a function of the separation between source and detector fibers in the optical probe. The depth of the tissue interrogated by the broad-spectrum source is approximately one-third to one-half of the distance between the source and detector fiber. Thus, progressively deeper tissue is probed by each detector that is positioned further from the source fiber in the optical probe.

It is of interest to measure depth dependencies in tissue oxygenation because tumors are heterogeneously vascularized and/or oxygenated, including along differentials that exist as a function of their depth (28). Thus, we next considered if DRS could detect any depth-dependent differences in lesion oxygenation. DRS data were separated into that collected by detectors at positions of $0.14,0.20,0.28$ and $0.34 \mathrm{~cm}$ from the source fiber, 
a
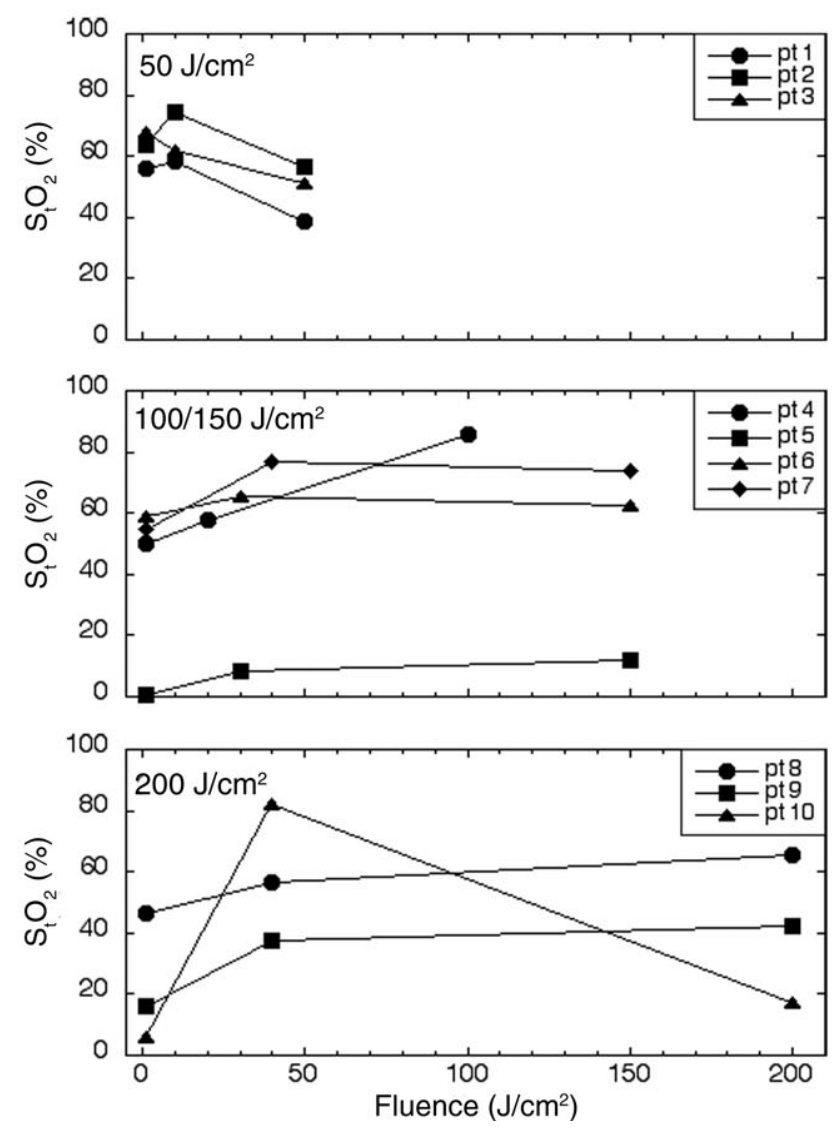

b
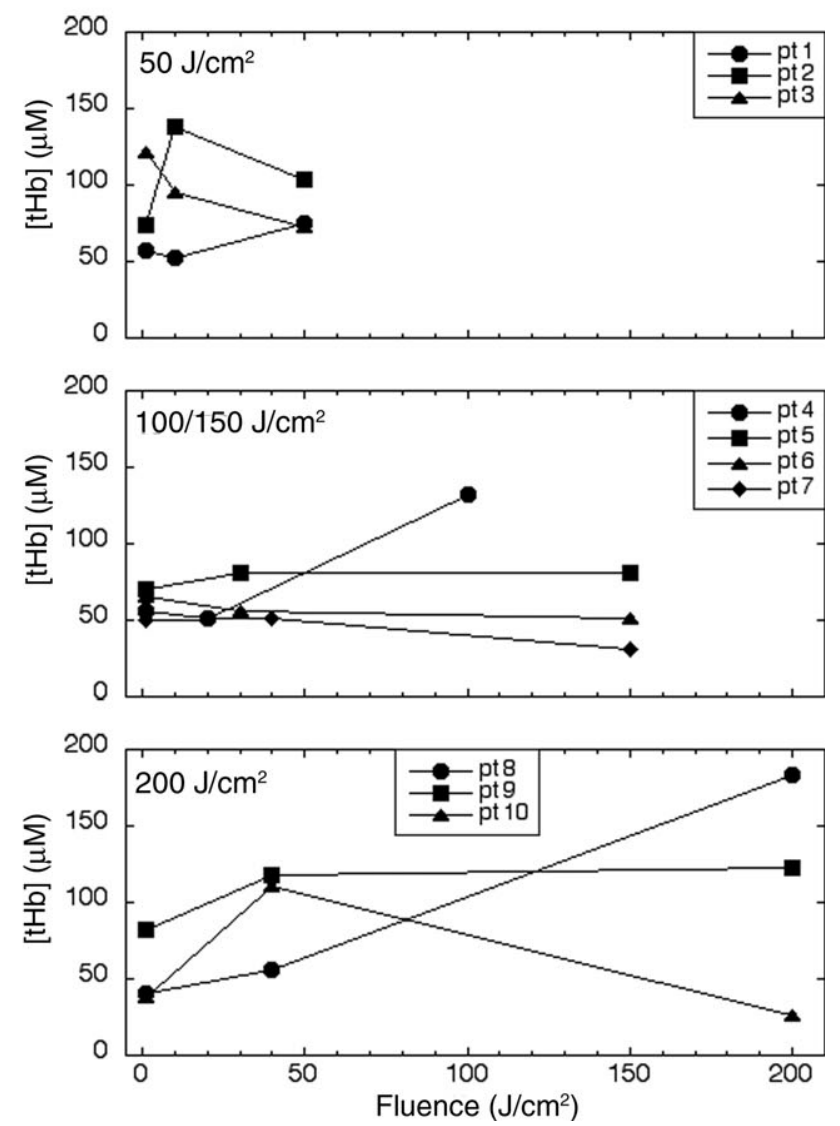

Figure 3. (a) Lesion $\mathrm{S}_{\mathrm{t}} \mathrm{O}_{2}$ and (b) [tHb] measured at baseline $\left(0 \mathrm{~J} \mathrm{~cm}^{-2}\right.$, first plotted point), during the dark interval of fractionation (second plotted point) and at treatment conclusion (third plotted point). Lesions of the oral cavity were illuminated at $632 \mathrm{~nm}$ at $4-6 \mathrm{~h}$ after oral ingestion of ALA. Illumination to the indicated light fluence was fractionated by a 90-180 s interruption of light delivery after $20 \%$ of the fluence. For clarity, panels separate patients who were treated at different fluences.

subsequently called positions $\mathrm{A}, \mathrm{B}, \mathrm{C}$ and $\mathrm{D}$ on the probe (see Fig. 1 for schematic). Based on a depth probed that is $\sim 50 \%$ of the source-detector separation, the depth of tissue probed ranged from the most superficial $0.07 \mathrm{~cm}$ of the lesion (at position A) to within $0.17 \mathrm{~cm}$ of the lesion surface (at position D). These measurement depths are well matched to the lesions of this study, which by definition were $0.15 \mathrm{~cm}$ or less in depth excluding limited microfocal invasion of the basement membrane. Figure $4 \mathrm{a}$ summarizes depth-dependent differences in lesion $\mathrm{S}_{\mathrm{t}} \mathrm{O}_{2}$ as a function of source-detector separation. Paired differences in median $\mathrm{S}_{\mathrm{t}} \mathrm{O}_{2}$ values were consistently positive when comparing more superficial to deeper detectors. At the most superficial pairing (A versus B), the median difference of $6.1 \%$ did not achieve statistical significance. At the more distant pairings of $\mathrm{A}$ with $\mathrm{C}$ and $\mathrm{A}$ with $\mathrm{D}$, lesion oxygenation was significantly higher at the lesion's surface (respective median differences of $14.4 \%$ and $15.8 \%, P<0.05$ for both pairings). Similarly, at intermediate depth the pairing of $\mathrm{B}$ with $\mathrm{C}$ (median difference of $10.0 \%, P=0.014$ ) was statistically significant for higher oxygenation in the more superficial measurement; the pairing of $\mathrm{B}$ with $\mathrm{D}$ was of a similar magnitude (median difference of $14.3 \%$ ), but did not achieve strict statistical significance $(P=0.105)$. At the deepest regions probed, the median difference in lesion oxygenation was only $2.3 \%$ between the fibers in the $\mathrm{C}$ and $\mathrm{D}$ positions.

These differences in $\mathrm{S}_{\mathrm{t}} \mathrm{O}_{2}$ are characteristic of the lesions being measured. In contrast, normal tissue of the oral cavity exhibits less variation with depth, as shown in Fig. 4b. The median paired difference ranged from -3.0 to $10.9 \%$ with only one paired difference exceeding $10 \%$. Only at the most superficial depth comparison (A versus B) was the difference statistically significant $(P=0.049$, median difference of $8.5 \%)$, which is consistent with the oxygenation of air-exposed mucosal and cutaneous surfaces through uptake of atmospheric oxygen $(29,30)$.

\section{Assessment of PpIX photobleaching}

In addition to physiologic properties, tumor levels of PpIX during the delivery of PDT are known to be predictive of treatment effect (31,32). We have measured sensitizer fluorescence using both the CFRS and NCTF systems. Typical fluorescence spectra taken using these systems are shown in Fig. 5a,b. The components of the fits to these data are also shown. The PpIX levels measured using the contact probe varied by over 10-fold among the lesions of different patients before PDT was begun (see Table 1). As expected, PDT did produce substantial changes in lesion PpIX levels. At treatment conclusion, most PpIX had photobleached in the lesions (Fig. 6a). Moreover, photobleaching occurred quickly, as low PpIX levels were detectable by the break in light delivery for fractionation. It is important to emphasize that for these measurements, only the most superficial layer of the lesions was probed because PpIX was determined by its fluorescence spectra following excitation at $403 \mathrm{~nm}$. Given this 
a

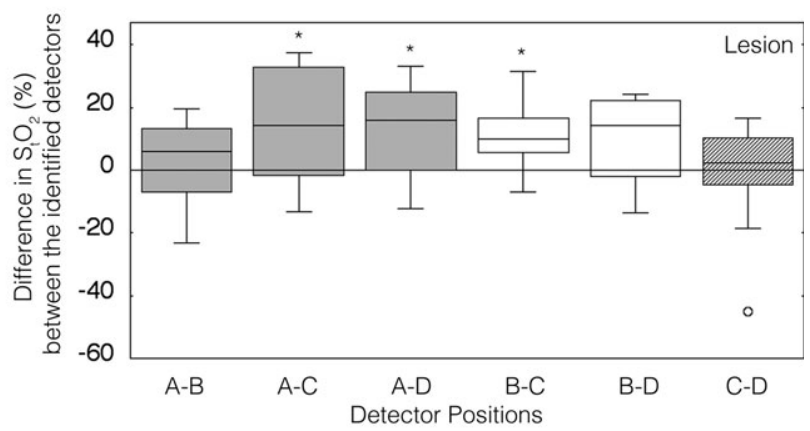

b

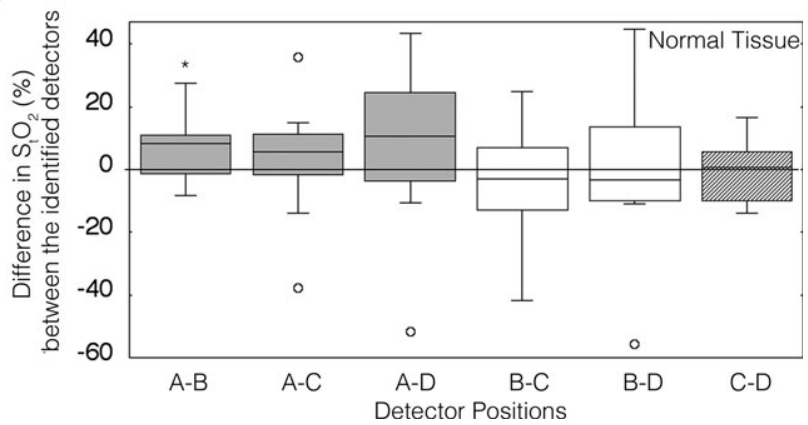

Figure 4. (a) Depth-dependent differences in the pre-PDT oxygenation of oral lesions and (b) normal tissues. Data plot the difference in tissue $\mathrm{S}_{\mathrm{t}} \mathrm{O}_{2}$ measured by discrete pairings of the source fiber with detector fibers in the DRS probe, thereby revealing differences in oxygenation between the tissue depth measured by each source-detector pair. For simplicity, detectors at a distance of $0.14,0.20,0.28$ and $0.34 \mathrm{~cm}$ from the source fiber are identified as positions A, B, C and D, respectively (see Fig. 1). Differences in $\mathrm{S}_{\mathrm{t}} \mathrm{O}_{2}$ between each pair of positions are plotted as boxplots with outliers identified as open circles. *Indicates $P<0.05$ for a nonzero depth-dependent difference.

wavelength of excitation, most of the detected signal originated from the tissue surface.

Using the treatment light as the excitation source with the NCTF system, it is possible to probe for PpIX fluorescence in deeper tissues on average than those sampled with the contact probe. For measurements that used the treatment light, the photobleaching time course, averaged over all patients at each fluence, is shown in Fig. 6b. In general, these data show that the deeper tissues bleach more slowly than the superficial layers, likely because they receive a lower fluence rate of light. For example, in patients treated at $50 \mathrm{~J} \mathrm{~cm}^{-2}$, relative PpIX level was $\sim 0.1$ at the tissue surface when illumination was paused at $10 \mathrm{~J} \mathrm{~cm}^{-2}$ for the dark interval of fractionation (Fig. 6a). This can be compared to that detected by more deeply probing measurements (Fig. 6b), for which a relative PpIX level of $\sim 0.5$ was found at the point of fractionation for $50 \mathrm{~J} \mathrm{~cm}^{-2}$ (i.e. $10 \mathrm{~J} \mathrm{~cm}^{-2}$ ). However, at the conclusion of the highest-light dose treatments, even the deeper layers probed by treatment light are almost completely photobleached.

\section{DISCUSSION}

In this study, we have demonstrated the feasibility of measuring diffuse reflectance and fluorescence spectra as part of a clinical protocol for superficial oral cavity lesions. The patient-to-patient variations in both pre-existing optical properties and sensitizer concentration and the changes induced by PDT were significant.

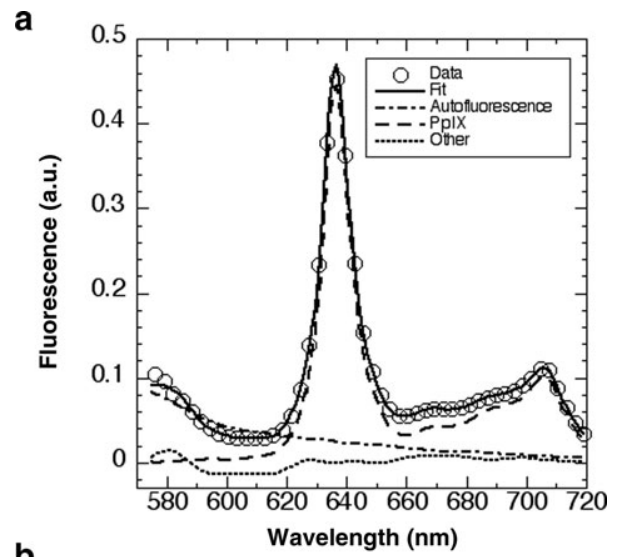

b

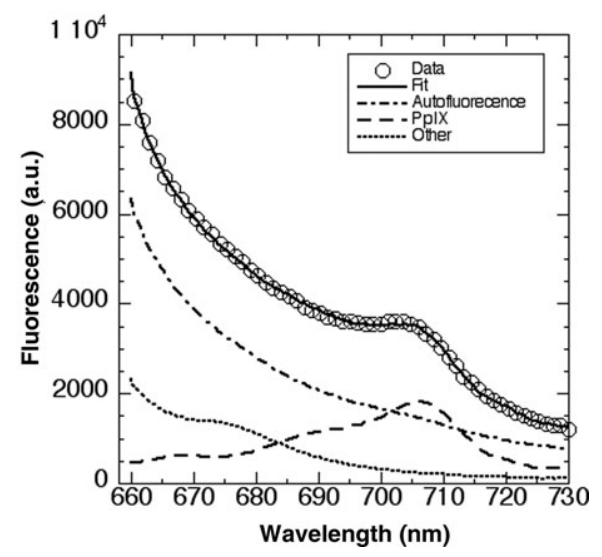

Figure 5. (a) Typical fluorescence spectrum measured using the CFRS system. The measured data are shown along with the fits and the contributions of PpIX, autofluorescence and the sum of the remaining fitting components. (b) Typical fluorescence spectrum excited by the treatment light and collected by the NCTF system. The contributions from various components are shown.

This has implications for patient-specific dosimetry. By prescribing a set measured fluence rate at the tissue surface, we use a patient-specific dosimetry protocol that inherently accounts for variations in patient geometry and optical properties and delivers a prescribed fluence rate to the target tissue. Despite this, we still see variations in response to PDT. Given the variation we see in sensitizer fluorescence, this is not surprising. The next order of refinement in patient-specific dosimetry could take one of two courses.

The explicit dosimetry approach (15) would measure the fluence rate of light, the sensitizer concentration and the tissue oxygenation. The dose would then be defined as the product of light fluence and sensitizer concentration or by the cumulative singlet oxygen concentration delivered to the tissue, as predicted by a suitable model $(33,34)$. Measurements such as those presented here could provide the inputs for such a model. Explicit PDT dosimetry is an area of ongoing investigation. The variability we see among lesions in this study suggests that different patients may require different fluence rates and different total fluences to achieve similar therapeutic effects.

Alternatively, an implicit dosimetry strategy would use a surrogate such as sensitizer photobleaching as an indicator of PDT dose. The implicit dosimetry approach is in principle simpler because it requires the measurement of only one parameter, the fraction of the sensitizer that has been bleached. Our NCTF 
a

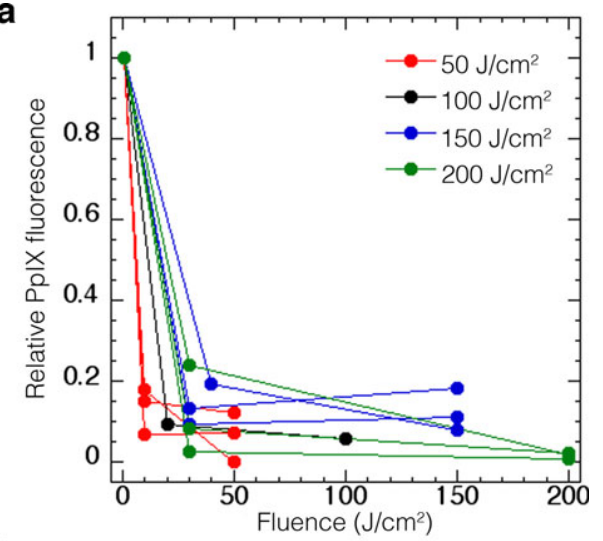

b

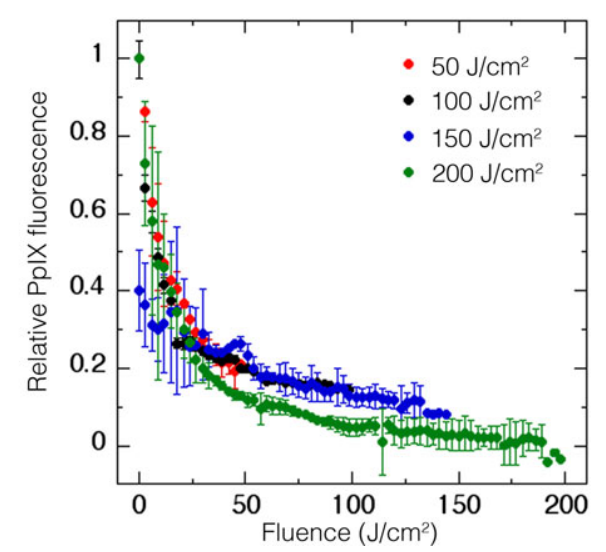

Figure 6. (a) Photobleaching of PpIX measured by the CFRS system (detector position $\mathrm{A})$ at baseline $\left(0 \mathrm{~J} \mathrm{~cm}^{-2}\right.$, first plotted point), during the dark interval of fractionation (second plotted point) and at treatment conclusion (third plotted point). Data are normalized to a value of 1 based on measurements made immediately before PDT (baseline); plots indicate individual patients. (b) Photobleaching of PpIX fluorescence collected by the NCTF system over the course of treatment. Measurements made through excitation by the treatment light (probing more deeply than the contact probe used to collect the data of panel (a). Data are normalized to a value of 1 at the start of treatment, and plotted as the average for each cohort. Error bars represent the standard deviation within each cohort.

measurements would provide the data needed to implement an implicit dosimetry protocol. Again, although PpIX was bleached in all cases, the variation in photobleaching rate indicates that following an implicit PDT protocol could result in different fluences and fluence rates for different patients.

Among these patients, lesion $\mathrm{S}_{\mathrm{t}} \mathrm{O}_{2}$ averaged $42 \pm 25 \%( \pm \mathrm{SD})$ at baseline. The majority of patients had values $>45 \%$, whereas three patients had baseline values of $<20 \%$. Based on the oxyhemoglobin dissociation curve, a blood oxyhemoglobin saturation of $50 \%$ is associated with an oxygen partial pressure of $\sim 27 \mathrm{mmHg}$. This is well above the half value of $7.6 \mathrm{mmHg}$ for PDT-created cytotoxicity (35). It is only in patients 5, 9 and 10 at baseline and patients 5 and 10 after PDT that lesion $\mathrm{S}_{\mathrm{t}} \mathrm{O}_{2}$ values corresponded to oxygen partial pressures of less than $\sim 15 \mathrm{mmHg}$, where limitations in PDT efficacy may come in to play. Interestingly, both patients 5 and 10 had a local recurrence at 3 months after PDT, which makes it of great interest to further study the association between lesion oxygenation and PDT outcomes.
The average baseline $\mathrm{S}_{\mathrm{t}} \mathrm{O}_{2}$ value of $42 \pm 25 \%$ was indistinguishable from an average value of $34 \pm 21 \%$ detected in the normal oral tissues (data not shown). Others have also reported the $\mathrm{S}_{\mathrm{t}} \mathrm{O}_{2}$ of normal mucosa and dysplastic lesions of the oral cavity to be similar, whereas oxygenation of the mucosa of oral squamous cell carcinoma is significantly lower $(36,37)$. In these studies, the average $\mathrm{S}_{\mathrm{t}} \mathrm{O}_{2}$ of oral tissue/cancer was between $\sim 80$ and $95 \%$, compared to the averages of $\sim 35-40 \%$ detectable in our study. This difference may be attributable to the design of the probes used for optical spectroscopy in each study. Our probe interrogated tissue up to $\sim 1.7 \mathrm{~mm}$ in depth (assuming a measurement volume that extends to roughly one-half of the maximum source-detector separation) and thus provides an average of the optical properties of tissues that include surface epithelial tissue through underlying stroma (38). In the study by Amelink et al. (37), the probe interrogated only within $\sim 350 \mu \mathrm{M}$ of the surface, (i.e. the epithelial layer), which was more superficial than that measured by even our most closely positioned source-detector pair (it measured to a depth $\sim 0.7 \mathrm{~mm}$ ). It is important to note here that we did detect depthdependent differences in lesion oxygenation showing that the more superficial layers of the lesion are better oxygenated, albeit the values did not reach the high $\mathrm{S}_{\mathrm{t}} \mathrm{O}_{2}$ percentages reported by Amelink et al.

Others have also reported that depth-dependent variation exists in the reflectance spectra of oral lesions (39). The authors of this study concluded that the spectra in dysplastic or cancerous tissue were more greatly affected by disease-induced differences in the light scattering properties of the epithelium than in deeper underlying tissue (39). They hypothesized that this was a result of changes in the nuclear composition of cells in the epithelium as a function of disease progression $(39,40)$. Moreover, the presence of overlying keratin on the lesion or keratin pearls deep within the epithelium, as is characteristic of oral (erythro) leukoplakia (40), could contribute to architectural changes that locally alter tissue physiology as detected by spectroscopy (36). Overlying keratin could also explain the absence of an oxygen differential between the two most superficial measurements (AB) in the lesions of our study (contrasted to the presence of a differential in normal tissue). This follows the facts that keratin overlying a lesion could impede the transmucosal diffusion of oxygen and/or increase the distance between the mucosal layer and probe contact with the tissue surface.

PDT led to both increases and decreases in lesion $\mathrm{S}_{\mathrm{t}} \mathrm{O}_{2}$ and [tHb] in this study. These physiologic effects were detectable both during the dark interval of fractionation and at treatment conclusion. Using continuous measurements of tissue $\mathrm{pO}_{2}$ during ALA-PDT of the rat colon, others have found that fractionation leads to recoveries of tissue oxygenation after its decrease at the initiation of light delivery (41). Also using continuous measurements, Pogue et al. (10) documented progressive increases in the $\mathrm{pO}_{2}$ of murine tumors during illumination for ALA-PDT that remained elevated immediately after light delivery. They attributed these increases to contributions from a reduction in metabolic oxygen consumption secondary to PDT-induced cell death and/or PDT-created vascular effects. Yet, others have documented vasoconstriction to take place with PDT using topically applied ALA $(42,43)$, and both measurements and modeling suggest that ALA-PDT can reduce local blood flow $(44,45)$. Ultimately, vascular effects during illumination for ALA-PDT are highly dynamic (27), and the resulting oscillations in blood flow 
make it quite possible that patient-to-patient differences will exist in physiologic parameters for measurements made at a single timepoint. Indeed, a clinical study of ALA-PDT in patients with skin carcinoma (basal cell or squamous) documented variable PDT effect on hemoglobin concentration (46). This result is not unlike that which we have detected in this study. Overall, the data from this trial are consistent with the possibility that lesion physiologic status after PDT is determined by various factors, such as PDT-induced ischemia or changes in oxygen consumption, and the balance of the contributions by these factors will likely vary among patients. Together with the above-described studies, these data reaffirm the need to measure physiologic parameters on an individual basis in patients.

Because of the potential for tumor concentrations of PpIX to contribute to physiologic responses to PDT, we also evaluated pre-existing and PDT effect on PpIX levels. Photobleaching produced very clear trends in PpIX levels during PDT that were independent of physiologic properties or initial PpIX level. Most photobleaching within the surface of the lesion (measured by a source-detector separation of $0.14 \mathrm{~cm}$ using $405 \mathrm{~nm}$ illumination) occurred by the break for fractionation. These findings align well with multiple other investigations that establish PpIX to be rapidly photobleached by PDT $(17,18,47)$. Nevertheless, it is interesting that most photobleaching occurred by the time of fractionation irrespective of the fluence delivered. Although this suggests that the continued delivery of light may provide little additional therapeutic benefit, it is crucial to remember that these measurements reflect only the superficial photobleaching. Indeed, in deeper tissue layers that were measured during the course of illumination, photobleaching was detected throughout light delivery, with complete photobleaching reached near the conclusion of the high fluence treatments. Thus, there is reason to expect that in deeper layers the potential for a cytotoxic effect is maintained throughout the treatment course.

In conclusion, this study demonstrates the feasibility of making both contact and noncontact spectroscopic measurements during PDT in the clinic, and also point out some key advantages and disadvantages of each. Noncontact measurements have the advantage that they can be made continuously during treatment, and potentially used for dosimetry. Because they use treatment light to excite fluorescence, the region sampled by the fluorescence measurements is closer to the volume being treated than in the case of fluorescence excited by shorter wavelengths. On the other hand, the CFRS system provides a wealth of data extracted from diffuse reflectance measurement that is not available in the noncontact measurements. Ultimately, both measurements could be useful in clinical implementation of personalized PDT dosimetry. The CFRS system, or one with similar capabilities, could be used to characterize the lesion before treatment and assess the concentration of sensitizer and physiologic abnormalities that might influence the selection of prescribed light dose. Ideally, this measurement would be performed in an imaging mode, rather than point-by-point. The technology to accomplish this has been demonstrated in preclinical models $(48,49)$. Then, the NCTF system could be used to monitor photobleaching during treatment to confirm that the prescribed dose is achieving the intended biological effect. The present manuscript establishes the clinical feasibility and utility of rationally designed spectroscopy probes that can be employed toward personalized dosimetry in PDT of the oral cavity, as well as at other potential treatment sites.
Acknowledgements-This work was supported in part by the National Institutes of Health (CA-129554, CA-087971, CA-085831 and T32-CA009677). The authors thank DUSA Pharmaceuticals, Inc, for providing the study drug. We also acknowledge assistance from postdoctoral fellow Dr. Craig Grossman; clinical trial coordinator Michael Mehler; laser specialist Carmen Rodriguez and research nurses Deborah Smith and Sally McNulty.

\section{REFERENCES}

1. Sunar, U. (2013) Monitoring photodynamic therapy of head and neck malignancies with optical spectroscopies. World J. Clin. Cases 1, 96-105.

2. Quon, H., C. E. Grossman, J. C. Finlay, T. C. Zhu, C. S. Clemmens, K. M. Malloy and T. M. Busch (2011) Photodynamic therapy in the management of pre-malignant head and neck mucosal dysplasia and microinvasive carcinoma. Photodiagnosis Photodyn. Ther. 8, 75-85.

3. Jemal, A., F. Bray, M. M. Center, J. Ferlay, E. Ward and D. Forman (2011) Global cancer statistics. CA Cancer J. Clin. 61, 69-90.

4. (based on November 2013 SEER data submission, posted to the SEER web site, April 2014.) SEER Cancer Statistics Review, 19752011. Available at: http://seer.cancer.gov/csr/1975_2011/. Accessed on 23 April 2014.

5. Biel, M. A. (2010) Photodynamic therapy of head and neck cancers. Methods Mol. Biol. 635, 281-293.

6. Agostinis, P., K. Berg, K. A. Cengel, T. H. Foster, A. W. Girotti, S. O. Gollnick, S. M. Hahn, M. R. Hamblin, A. Juzeniene, D. Kessel, M. Korbelik, J. Moan, P. Mroz, D. Nowis, J. Piette, B. C. Wilson and J. Golab (2011) Photodynamic therapy of cancer: an update. $C A$ Cancer J. Clin. 61, 250-281.

7. Busch, T. M. (2006) Local physiological changes during photodynamic therapy. Lasers Surg. Med. 38, 494-499.

8. Milstein, D. M., A. M. van Kuijen, M. P. Copper, B. Karakullukcu, I. B. Tan, J. A. Lindeboom, W. J. Fokkens and C. Ince (2012) Monitoring microcirculatory alterations in oral squamous cell carcinoma following photodynamic therapy. Photodiagnosis Photodyn. Ther. 9, 69-75.

9. Wang, H. W., M. E. Putt, M. J. Emanuele, D. B. Shin, E. Glatstein, A. G. Yodh and T. M. Busch (2004) Treatment-induced changes in tumor oxygenation predict photodynamic therapy outcome. Cancer Res. 64, 7553-7561.

10. Pogue, B. W., J. A. O'Hara, I. A. Goodwin, C. J. Wilmot, G. P. Fournier, A. R. Akay and H. Swartz (2002) Tumor PO(2) changes during photodynamic therapy depend upon photosensitizer type and time after injection. Comp. Biochem. Physiol. A Mol. Integr. Physiol. 132, 177-184.

11. Wilson, B. and M. Patterson (2008) The physics, biophysics and technology of photodynamic therapy. Phys. Med. Biol. 53, 61-109.

12. Finlay, J. C. and T. H. Foster (2004) Hemoglobin oxygen saturations in phantoms and in vivo from measurements of steady-state diffuse reflectance at a single, short source-detector separation. Med. Phys. 31, 1949-1959.

13. Dong, J., H. J. Toh, P. S. Thong, C. S. Tee, R. Bi, K. C. Soo and K. Lee (2014) Hemodynamic monitoring of Chlorin e6-mediated photodynamic therapy using diffuse optical measurements. J. Photochem. Photobiol. B 140, 163-172.

14. Zhou, X., B. W. Pogue, B. Chen, E. Demidenko, R. Joshi, J. Hoopes and T. Hasan (2006) Pretreatment photosensitizer dosimetry reduces variation in tumor response. Int. J. Radiat. Oncol. Biol. Phys. 64, 1211-1220.

15. Wilson, B., M. Patterson and L. Lilge (1997) Implicit and explicit dosimetry in photodynamic therapy: a new paradgm. Lasers Surg. Med. 12, 182-199.

16. Robinson, D. J., H. S. de Bruijn, N. van der Veen, M. R. Stringer, S. B. Brown and W. M. Star (1998) Fluorescence photobleaching of ALA-induced protoporphyrin IX during photodynamic therapy of normal hairless mouse skin: the effect of light dose and irradiance and the resulting biological effect. Photochem. Photobiol. 67, 140-149.

17. Finlay, J. C., D. L. Conover, E. L. Hull and T. H. Foster (2001) Porphyrin bleaching and PDT-induced spectral changes are irradiance dependent in ALA-sensitized normal rat skin in vivo. Photochem. Photobiol. 73, 54-63. 
18. Cottrell, W. J., A. D. Paquette, K. R. Keymel, T. H. Foster and A. R. Oseroff (2008) Irradiance-dependent photobleaching and pain in delta-aminolevulinic acid-photodynamic therapy of superficial basal cell carcinomas. Clin. Cancer Res. 14, 4475-4483.

19. Finlay, J. C., T. C. Zhu, A. Dimofte, D. Stripp, S. B. Malkowicz, T. M. Busch and S. M. Hahn (2006) Interstitial fluorescence spectroscopy in the human prostate during motexafin lutetium-mediated photodynamic therapy. Photochem. Photobiol. 82, 1270-1280.

20. Zhu, T. C., J. C. Finlay and S. M. Hahn (2005) Determination of the distribution of light, optical properties, drug concentration, and tissue oxygenation in-vivo human prostate during motexafin luteium-mediated photodynamic therapy. J. Photochem. Photobiol. B 79, 231241.

21. Wang, H.-W., J. C. Finlay, K. Lee, T. C. Zhu, M. E. Putt, E. Glatstein, C. J. Koch, S. M. Evans, S. M. Hahn, T. M. Busch and A. G. Yodh (2007) Quantitative comparison of tissue oxygen and motexafin lutetium uptake by ex vivo and noninvasive in vivo techniques in patients with intraperitoneal carcinomatosis. J. Biomed. Opt. 12, 034023.

22. Sharikova, A. V., J. C. Finlay, X. Liang and T. C. Zhu (2013) PDT dose dosimetry for pleural photodynamic therapy. Proc. SPIE. 8568, pii: 856817.

23. Grossman, C., T. Zhu, J. Finlay, A. Dimofte, K. Malloy, B. Jr O'Malley, G. Weinstein, T. M. Busch and H. Quon (2010) Targeted laryngeal photodynamic therapy with a balloon diffusing light source. Photodiagnosis Photodyn. Ther. 7, 158-161.

24. Curnow, A., B. W. McIlroy, M. J. Postle-Hacon, A. J. MacRobert and S. G. Bown (1999) Light dose fractionation to enhance photodynamic therapy using 5-aminolevulinic acid in the normal rat colon. Photochem. Photobiol. 69, 71-76.

25. Mourant, J. R., T. Fuselier, J. Boyer, T. Johnson and I. J. Bigio (1997) Predictions and measurements of scattering and absorption over broad wavelength ranges in tissue phantoms. Appl. Opt. 36, 948-957.

26. Hull, E., M. G. Nichols and T. Foster (1998) Quantitative broadband near infrared spectroscopy of tissue-simulating phantoms containing erythrocytes. Phys. Med. Biol. 43, 3381-3404.

27. Becker, T. L., A. D. Paquette, K. R. Keymel, B. W. Henderson and U. Sunar (2010) Monitoring blood flow responses during topical ALA-PDT. Biomed. Opt. Express 2, 123-130.

28. Dome, B., S. Paku, B. Somlai and J. Timar (2002) Vascularization of cutaneous melanoma involves vessel co-option and has clinical significance. J. Pathol. 197, 355-362.

29. Stucker, M., A. Struk, P. Altmeyer, M. Herde, H. Baumgartl and D. W. Lubbers (2002) The cutaneous uptake of atmospheric oxygen contributes significantly to the oxygen supply of human dermis and epidermis. J. Physiol. 538, 985-994.

30. Hamada, Y., H. Utahashi and K. Aoki (2002) Physiological gas exchange in the middle ear cavity. Int. J. Pediatr. Otorhinolaryngol. 64, 41-49.

31. Mallidi, S., S. Anbil, S. Lee, D. Manstein, S. Elrington, G. Kositratna, D. Schoenfeld, B. Pogue, S. J. Davis and T. Hasan (2014) Photosensitizer fluorescence and singlet oxygen luminescence as dosimetric predictors of topical 5-aminolevulinic acid photodynamic therapy induced clinical erythema. J. Biomed. Opt. 19, 028001.

32. Tyrrell, J. S., S. M. Campbell and A. Curnow (2010) The relationship between protoporphyrin IX photobleaching during real-time dermatological methyl-aminolevulinate photodynamic therapy (MALPDT) and subsequent clinical outcome. Lasers Surg. Med. 42, 613619.

33. Wang, K. K.-H., J. Finlay, T. M. Busch, S. Hahn and T. C. Zhu (2010) Explicit dosimetry for photodynamic therapy: macroscopic singlet oxygen modeling. J. Biophotonics 3, 304-318.
34. Wang, K. K.-H., S. Mitra and T. H. Foster (2008) Photodynamic dose does not correlate with long-term tumor response to mTHPCPDT performed at several drug-light intervals. Med. Phys. 35, 3518.

35. Henderson, B. W. and V. H. Fingar (1987) Relationship of tumor hypoxia and response to photodynamic treatment in an experimental mouse tumor. Cancer Res. 47, 3110-3114.

36. Amelink, A., H. J. Sterenborg, J. L. Roodenburg and M. J. Witjes (2011) Non-invasive measurement of the microvascular properties of non-dysplastic and dysplastic oral leukoplakias by use of optical spectroscopy. Oral Oncol. 47, 1165-1170.

37. Amelink, A., O. P. Kaspers, H. J. Sterenborg, J. E. van der Wal, J. L. Roodenburg and M. J. Witjes (2008) Non-invasive measurement of the morphology and physiology of oral mucosa by use of optical spectroscopy. Oral Oncol. 44, 65-71.

38. Schwarz, R. A., W. Gao, D. Daye, M. D. Williams, R. RichardsKortum and A. M. Gillenwater (2008) Autofluorescence and diffuse reflectance spectroscopy of oral epithelial tissue using a depth-sensitive fiber-optic probe. Appl. Opt. 47, 825-834.

39. Schwarz, R. A., W. Gao, C. Redden Weber, C. Kurachi, J. J. Lee, A. K. El-Naggar, R. Richards-Kortum and A. M. Gillenwater (2009) Noninvasive evaluation of oral lesions using depth-sensitive optical spectroscopy. Cancer 115, 1669-1679.

40. Speight, P. M. (2007) Update on oral epithelial dysplasia and progression to cancer. Head Neck Pathol. 1, 61-66.

41. Curnow, A., J. C. Haller and S. G. Bown (2000) Oxygen monitoring during 5-aminolaevulinic acid induced photodynamic therapy in normal rat colon. Comparison of continuous and fractionated light regimes. J. Photochem. Photobiol. B 58, 149-155.

42. van Leeuwen-van Zaane, F., H. S. de Bruijn, A. van der Ploeg-van den Heuvel, H. J. Sterenborg and D. J. Robinson (2014) The effect of fluence rate on the acute response of vessel diameter and red blood cell velocity during topical 5-aminolevulinic acid photodynamic therapy. Photodiagnosis Photodyn. Ther. 11, 71-81.

43. Middelburg, T. A., H. S. de Bruijn, L. Tettero, A. van der Ploeg van den Heuvel, H. A. Neumann, E. R. de Haas and D. J. Robinson (2013) Topical hexylaminolevulinate and aminolevulinic acid photodynamic therapy: complete arteriole vasoconstriction occurs frequently and depends on protoporphyrin IX concentration in vessel wall. J. Photochem. Photobiol. B 126, 26-32.

44. Wang, K. K., W. J. Cottrell, S. Mitra, A. R. Oseroff and T. H. Foster (2009) Simulations of measured photobleaching kinetics in human basal cell carcinomas suggest blood flow reductions during ALA-PDT. Lasers Surg. Med. 41, 686-696.

45. Schacht, V., R. M. Szeimies and C. Abels (2006) Photodynamic therapy with 5 -aminolevulinic acid induces distinct microcirculatory effects following systemic or topical application. Photochem. Photobiol. Sci. 5, 452-458.

46. Johansson, A., T. Johansson, M. S. Thompson, N. Bendsoe, K. Svanberg, S. Svanberg and S. Andersson-Engels (2006) In vivo measurement of parameters of dosimetric importance during interstitial photodynamic therapy of thick skin tumors. J. Biomed. Opt. 11, 34029.

47. Robinson, D. J., H. S. de Bruijn, N. van der Veen, M. R. Stringer, S. B. Brown and W. M. Star (1999) Protoporphyrin IX fluorescence photobleaching during ALA-mediated photodynamic therapy of UVB-induced tumors in hairless mouse skin. Photochem. Photobiol. 69, 61-70.

48. Sunar, U., D. J. Rohrbach, J. Morgan, N. Zeitouni and B. W. Henderson (2013) Quantification of PpIX concentration in basal cell carcinoma and squamous cell carcinoma models using spatial frequency domain imaging. Biomed. Opt. Express 4, 531-537.

49. Konecky, S. D., C. M. Owen, T. Rice, P. A. Valdés, K. Kolste, B. C. Wilson, F. Leblond, D. W. Roberts, K. D. Paulsen and B. J. Tromberg (2012) Spatial frequency domain tomography of protoporphyrin IX fluorescence in preclinical glioma models. J. Biomed. Opt. 17, 056008. 